\title{
Optimal Debulking in Advanced Epithelial Ovarian Cancer \& Its Achievable Outcome
}

\author{
NEERJA BHATLA, RAJNI BANSAL, DEEPA MAHESWARI
}

Ovarian cancer is the third most common cause of death from cancer among women in India. Most women present in Stage III of the disease. Fewer than 20 percent of patients with advanced cancer are alive five years after the diagnosis. One of the important reasons for this is the inability to perform optimal debulking in these cases.

Optimal surgical status is currently defined as residual disease $\leq 1 \mathrm{~cm}$. The goal of surgery for ovarian cancer is total abdominal hysterectomy with bilateral salpingo-oophorectomy and infracolic omentectomy and maximal surgical effort at cytoreduction. In advanced cases this may include appendectomy, resection of small and large bowel, spleen, liver, diaphragm, etc. However, the morbidity increases considerably with extensive surgery. Neoadjuvant chemotherapy has proved to be a boon for the surgeon as it enables shrinkage of the tumour and allows this goal to be achieved in a higher number of cases with less intra and postoperative morbidity.

Studies have reviewed the performance of women and assessed various parameters including age at diagnosis, performance status, histologic subtype, tumour grade, ascites volume, predominant peritoneal tumour pattern, surgical procedures performed, hepatic tumour residuum, extrahepatic tumour residuum, and postoperative complications. It was seen that overall median survival was 18.1 months and was highly correlated with performance status ( $P=0.002)$, predominant peritoneal tumour pattern $(P=0.0002)$, and the number of chemotherapy regimens received $(P=0.0039)$. When primary surgical cytoreduction was attempted in all patients, $30 \%$ achieved optimal status. Median survival of optimally cytoreduced patients was 38.4 months, compared to 10.3 months for patients with suboptimal residual disease $(P=0.0004)$.

Rates of optimal debulking surgery will vary depending on the type of institution and infrastructure, training and approach of the surgeon and general condition of the patient. Performance of these extensive procedures requires a committed and skilled team of doctors and paramedical support staff, adequate facilities for blood transfusion and the availability of an intensive care unit for postoperative recovery. However, optimal debulking surgery is an achievable goal which, along with performance status, is a significant predictor of survival in advanced ovarian cancer. 